\title{
MEDICIÓN DE LA ACTIVIDAD FÍSICA MEDIANTE ACELERÓMETROS TRIAXIALES EN ESCOLARES DE TRES CIUDADES DEL PERÚ
}

\author{
Katherine Alvis-Chirinos ${ }^{1, a}$, Lucio Huamán-Espino ${ }^{1, b}$, Jenny Pillaca ${ }^{1, c}$, Juan Pablo Aparco ${ }^{1, a}$
}

\begin{abstract}
RESUMEN
Objetivos. Determinar la proporción de escolares de educación primaria que cumplen la recomendación de la Organización Mundial de la Salud (OMS) sobre actividad física y los factores asociados a menor tiempo de actividad física moderada intensa en zonas urbanas de tres ciudades del Perú. Materiales y métodos. Estudio transversal con selección aleatoria de escolares de 6 a 13 años de instituciones educativas públicas de zonas urbanas de los distritos de Chiclayo, Huaraz y Tarapoto. Para medir el tiempo y la intensidad de la actividad física en los escolares se les puso un acelerómetro durante una semana, además se evaluó el estado nutricional a través del cálculo del índice de masa corporal. Resultados. Participaron 1241 escolares de ambos sexos, con edades entre 6 a 13 años. El $40 \%$ de escolares en Chiclayo, $56 \%$ en Tarapoto y $66 \%$ en Huaraz cumplían la recomendación de OMS de realizar como mínimo 60 minutos de actividad física moderada intensa al día. La intensidad de la actividad física fue significativamente mayor en los escolares de menor edad y los de sexo masculino. Además se encontró que los factores asociados a menos minutos de actividad física moderada intensa al día fueron escolares de mayor edad, de sexo femenino, con obesidad, con madres de nivel educativo superior y residentes en Chiclayo o Tarapoto $(p<0,05)$. Conclusiones. Cerca del $50 \%$ de escolares cumple la recomendación de OMS sobre actividad física diaria, los grupos menos activos son los escolares de mayor edad, obesos y de sexo femenino, por lo que se debe focalizar en ellos acciones para promover hábitos adecuados de actividad física.
\end{abstract}

Palabras clave: Actividad física; Acelerometría; Salud escolar; Estado nutricional. (Fuente: DeCS BIREME).

\section{MEASUREMENT OF PHYSICAL ACTIVITY BY TRIAXIAL ACCELEROMETERS IN SCHOOLCHILDREN FROM THREE PERUVIAN CITIES}

\begin{abstract}
Objectives. To determine the proportion of primary school students who fulfill the World Health Organization (WHO) recommendations on physical activity and the factors associated with less time of moderate-intense physical activity in urban areas of three cities in Peru. Materials and methods. Cross-sectional study with a random selection of schoolchildren aged 6 to 13 from public educational institutions in urban areas of Chiclayo, Huaraz and Tarapoto. In order to measure the time and intensity of physical activity, schoolchildren were given an accelerometer for a week. Furthermore, nutritional conditions were evaluated through the calculation of their body mass index. Results. A total of 1241 schoolchildren of both sexes aged between 6 and 13 years participated. $40 \%$ of schoolchildren in Chiclayo, $56 \%$ in Tarapoto and $66 \%$ in Huaraz fulfilled the WHO recommendations to perform at least 60 minutes of moderate-intense physical activity per day. The intensity of physical activity was significantly higher in younger schoolchildren and males. It was also found that the factors associated with fewer minutes of moderate-intense physical activity per day were schoolchildren of older age, females, with an obesity condition, whose mothers had higher educational level, and lived in Chiclayo or Tarapoto $(\mathrm{p}<0.05)$. Conclusions. Nearly $50 \%$ of schoolchildren meet the WHO recommendation on daily physical activity, the less active groups were older schoolchildren, obese and female; therefore, actions should be focused on them to promote adequate physical activity habits.
\end{abstract}

Key words: Physical activity; Accelerometry; School health; Nutritional status. (Source: MeSH NLM).

\footnotetext{
Centro Nacional de Alimentación y Nutrición, Instituto Nacional de Salud. Lima. Perú.

Nutricionista; ${ }^{\mathrm{b}}$ sociólogo; ${ }^{\mathrm{c}}$ estadístico

Recibido: 18/10/2016 Aprobado: 22/02/2017 En línea: 23/03/2017
}

Citar como: Alvis-Chirinos K, Huamán-Espino L, Pillaca J, Aparco JP. Medición de la actividad física mediante acelerómetros triaxiales en escolares de tres ciudades del Perú. Rev Peru Med Exp Salud Publica. 2017;34(1):28-35. doi: 10.17843/rpmesp.2017.341.2764 


\section{INTRODUCCIÓN}

La actividad física (AF) es uno de los principales factores que condicionan la salud humana, de tal modo que la inactividad física o sedentarismo constituye el cuarto factor de riesgo más importante de mortalidad en todo el mundo (1). Existe consenso sobre los beneficios de la $\mathrm{AF}$ en la salud y calidad de vida. En los adultos la AF reduce de 20 a $35 \%$ el riesgo de padecer enfermedades cardiovasculares (ECV), coronarias e infartos; previene la diabetes tipo II, el síndrome metabólico, incrementa la masa y fuerza muscular esquelética así como la activación neuromuscular ${ }^{(2)}$. Mientras que en los niños sus beneficios actúan sobre los procesos de crecimiento y desarrollo, y la prevención de factores de riesgo de ECV.

La AF se define como cualquier movimiento corporal que resulta en gasto energético en comparación al reposo ${ }^{(3)}$. La evidencia indica que los beneficios para la salud dependen del tipo y la intensidad de la AF. Por tanto, su medición precisa permite evaluar dimensiones complejas como el tipo, duración, frecuencia, intensidad ${ }^{(4)}$ e identifica a largo plazo, los patrones habituales.

La evidencia científica muestra consistentemente la relación entre $A F$ y salud ${ }^{(5)}$; sin embargo, la mayor parte de los estudios se basan en métodos de medición subjetivos mediante el uso de encuestas que miden la autopercepción que tiene el sujeto de la intensidad y duración de la actividad que realiza. El uso de estos métodos aumenta las diferencias relacionadas con la edad y sexo (6) a la vez que sobreestiman el tiempo de actividad en comparación con otros métodos objetivos.

La AF representa comportamientos complejos, por lo tanto, su medición también es propensa a tener errores a partir de las variaciones por cada día, la memoria y la estimación inexacta, sobre todo resulta difícil medir actividades moderadas que son parte de la rutina diaria ${ }^{(7)}$. Para evitar estos errores inherentes a la medición subjetiva es necesaria la obtención de parámetros que midan la AF en unidades cuantificables.

En la actualidad, para la medición objetiva de AF existen instrumentos conocidos como acelerómetros que son portátiles, con alto nivel de fiabilidad, y que han sido validados en poblaciones pediátricas. Estos instrumentos son fáciles de utilizar (es factible su uso en muestras grandes). Los acelerómetros miden el recuento de actividad, el gasto energético, la intensidad de la AF, la posición del cuerpo y la cantidad de sueño; basados en la medición del ritmo y magnitud con el que el centro de gravedad corporal se desplaza durante el movimiento ${ }^{(8)}$

En el Perú, la información sobre la AF es limitada, su cuantificación se ha realizado mediante métodos subjetivos, existen estudios realizados en escolares de nivel primario en Lima, Callao y Arequipa ${ }^{(9,10,11)}$.
En este contexto, donde la información sobre AF en escolares es insuficiente, surge la necesidad de generar evidencia usando métodos objetivos confiables. Los resultados de este estudio buscan contribuir a la implementación de acciones para mejorar la AF en la escuela, el hogar, los espacios públicos y brindar recomendaciones nutricionales más precisas. Desde esa perspectiva el estudio tiene como objetivo determinar la proporción de escolares de educación primaria que cumplen la recomendación de OMS sobre actividad física y los factores asociados a menor tiempo de actividad física moderada intensa en zonas urbanas de tres ciudades del Perú.

\section{MATERIALES Y MÉTODOS}

\section{DISEÑO DEL ESTUDIO}

Estudio de tipo descriptivo y transversal, realizado en escolares de zonas urbanas de tres ciudades de costa, sierra y selva de Perú.

\section{POBLACIÓN Y MUESTRA}

La población de estudio estuvo conformada por escolares de 6 a 13 años de edad, matriculados durante el 2014 en instituciones educativas (IE) públicas, de nivel primario, mixtos y de zonas urbanas. Se seleccionaron las regiones de Ancash, Lambayeque, San Martin porque presentaban indicadores de obesidad en escolares similares al promedio nacional. Dentro de cada región se seleccionaron los distritos con mayor número de alumnos; incluyéndose los distritos de Huaraz $(\mathrm{N}=4450)$, Chiclayo $(\mathrm{N}=16 \mathrm{988})$, y Tarapoto $(\mathrm{N}=6580)$.

El cálculo del tamaño de muestra se estimó de manera independiente para cada distrito, con una proporción esperada de $34 \%$ de escolares poco activos ${ }^{(11)}$, con un margen de error de $5 \%$, una tasa de no respuesta esperada del $10 \%$ y con un nivel de confianza del $95 \%$. El tamaño de muestra fue de 427 en Huaraz, 451 en Chiclayo y 437 en Tarapoto.

El muestreo fue probabilístico, bietápico, estratificado e independiente en cada una de las tres zonas. En la primera etapa de muestreo se seleccionaron instituciones educativas según probabilidad proporcional al tamaño, resultando 4 IE en Huaraz (de un total de 8), 4 IE en Tarapoto (de un total de 17) y 5 IE en Chiclayo (de un total de 39). En la segunda etapa se realizó un muestreo sistemático con arranque aleatorio, inicialmente en cada IE se elaboró un listado de alumnos elegibles por cada grado a partir de las nóminas de matrícula, luego se aplicaron los criterios de inclusión y exclusión y finalmente se extrajo la cuota asignada de escolares por cada grado. 
Se incluyeron escolares de seis a 13 años, con asistencia regular a clases (más de tres días en la semana anterior), con residencia urbana en el último año y con acuerdo de sus padres a participar en el estudio. Se excluyeron escolares con alguna enfermedad que pudiera interferir su nivel habitual de AF (invalidez física, síndrome de Down, cardiopatías, etc.) y aquellos que por motivos de festividades deportivas o celebraciones en la institución educativa realicen actividad física diferente de lo habitual.

\section{RECOLECCIÓN DE DATOS}

La recolección de datos se realizó en coordinación con la Dirección Regional de Educación (DRE) y Unidad de Gestión Educativa Local (UGEL), con participación de encuestadores a quienes se les capacito en la aplicación de la encuesta, en técnicas de evaluación antropométrica y en el manejo de acelerómetros. Dicho personal visitó las instituciones educativas, realizó la selección de estudiantes y las mediciones establecidas a los niños seleccionados.

La actividad física se midió con acelerómetros triaxiales (Actigraph GT3XBT+; Actigraph, LLC, Pensacola, $\mathrm{FL}$, USA), que recoge información de los tres ejes de movimientos (vertical, horizontal y perpendicular), con su respectivo software de procesamiento (Actilife versión 6.11.7). Se incluyeron datos de los escolares que tuvieron información como mínimo de 8 horas por día, entre las 6 a 22 horas, durante tres días. Se excluyeron del análisis los datos de periodos de 60 minutos continuos de recuento cero, considerando estos periodos como tiempos de no uso ${ }^{(12)}$. Se instruyó a padres y niños sobre el uso del acelerómetro. Se les indico retirárselo únicamente para bañarse, realizar actividades en contacto con el agua y para dormir durante la noche. Los dispositivos se sujetaron a la cintura con un cinturón elástico, hacia el lado derecho de cada niño quedando con el eje " $x$ " hacia arriba.

Para medir el estado nutricional, se tomaron datos antropométricos, siguiendo la técnica recomendada por el Centro Nacional de Alimentación y Nutrición (CENAN). Se utilizó un tallímetro de madera de tres cuerpos con una resolución de $0,1 \mathrm{~cm}$ y balanza electrónica marca Seca con resolución de $0,1 \mathrm{~kg}$. Con los datos de peso, talla y edad se calculó el Indice de Masa Corporal (IMC) para la edad, dividiendo el peso entre la talla al cuadrado. Al terminar la semana de medición con el dispositivo se aplicó un cuestionario para identificar otras características como nivel educativo de la madre, los periodos de no uso, los días escolares y con clase de educación física, así como un recordatorio de actividades durante un día que llevaron puesto el acelerómetro.

\section{VARIABLES E INDICADORES DEL ESTUDIO}

Para medir la AF, el acelerómetro utiliza las cuentas por minuto (cpm, counts per minute). Las cuentas son unidades que expresan cambios de aceleración en el cuerpo y son proporcionales a la intensidad de la AF, estos son obtenidos en un determinado intervalo de tiempo denominado epoch ${ }^{(13)}$. Además, el acelerómetro registra el tiempo en minutos de cada nivel de actividad física. Para categorizar la intensidad de AF se utilizó los puntos de corte de Evenson et al. ${ }^{(13)}$. De 0-100 cpm sedentarismo, de 101-2295 cpm actividad física (AF) ligera, de 2 296-4 $011 \mathrm{cp}$ m AF moderada y 4012 a más cpm AF vigorosa.

Para medir la actividad física moderada intensa (AFMI), se sumó los minutos del tiempo registrado como actividad moderada y actividad intensa. Se midió el cumplimiento de la recomendación OMS, considerando "Sí cumple" a los escolares que tuvieron un promedio de AFMI mayor o igual a 60 minutos al día ${ }^{(14)}$.

El estado nutricional, se midió con la clasificación del puntaje $z$ del IMC obtenido mediante el programa Anthro plus ${ }^{(15)}$, según los puntos de corte de la OMS ${ }^{(16)},<-2$ desviación estandar [DE] Delgadez, -2 a 1 DE Normal, $>1$ DE Sobrepeso y > 2 DE Obesidad. Para medir el nivel educativo de la madre se obtuvo información del ultimo nivel alcanzado según las siguientes categorías: Sin nivel/Inicial, Primaria, Secundaria y Superior Universitario/técnico.

\section{ANÁLISIS DE DATOS}

El procesamiento y análisis de la información se realizó en el programa Stata versión 12. Las variables cuantitativas se expresaron como mediana y rango intercuartilico y solo para el caso de cuentas por minutos de Tarapoto como media y DE; además, se utilizó la prueba de Shapiro Wilk para evaluar la normalidad de dichas variables. Las variables cualitativas se expresaron en frecuencias y porcentajes. Para evaluar las asociaciones entre las variables continuas se analizó el tipo de distribución de los datos y se aplicaron las pruebas $T$ de Student y $U$ de Mann Whitney en las diferencias según sexo y edad; ANOVA de una vía y $\mathrm{H}$ de Kruskal Wallis para la diferencia según estado nutricional. También se realizó un análisis de regresión lineal múltiple, utilizando información socioeconómica y biológica como variables independientes y como dependientes el tiempo de actividad moderada/intensa.

\section{ASPECTOS ETICOS}

El estudio fue evaluado y aprobado por el Comité de Ética del Instituto Nacional de Salud de Perú. Antes de realizar las mediciones se visitó los hogares de los niños seleccionados, y se explicó a los padres los objetivos, beneficios y riesgos 
de la participación en el estudio. Se obtuvo el consentimiento informado de los padres y el asentimiento informado de los escolares mayores de 9 años.

\section{RESULTADOS}

Se obtuvo información de 398 escolares de Huaraz, 416 de Chiclayo y 427 de Tarapoto, de 13 instituciones educativas públicas de nivel primario (4 en Huaraz, 4 en Tarapoto y 5 en Chiclayo). El promedio de edad fue 8,8 $(\mathrm{DE}=0,2)$ años en Huaraz; 8,7 ( $D E=0,1)$ en Chiclayo y $8,6$ ( $D E=0,1)$ años en Tarapoto. Las características de los escolares se detallan en la tabla 1, donde destacan ligeramente los varones en Chiclayo y las mujeres en Huaraz. En cuanto al estado nutricional, la misma tabla muestra que en Chiclayo se presentaron las mayores proporciones de escolares con sobrepeso y obesidad.

Respecto a la recomendación de la OMS de realizar 60 minutos de AFMI se encontró que en Chiclayo el $40,7 \%$ de escolares cumplía esta pauta, mientras que en Huaraz alcanzó al $66,6 \%$ de escolares y en Tarapoto fue de 56,4\%. La figura 1 muestra que el cumplimiento de la recomendación de la OMS, según sexo es significativamente diferente $(p<0,01)$ en los tres distritos. Según estado nutricional solo en Chiclayo se encontró diferencias entre las proporciones de escolares que cumplían la recomendación OMS $(p<0,01)$. Los escolares con obesidad cumplían menos la recomendación $(17,2 \%)$ que sus pares con sobrepeso $(40,6 \%)$ y estos a su vez cumplían menos que los de estado nutricional normal $(47,4 \%)$.

La figura 2 muestra la intensidad de AF, expresada en cuentas por minuto (cpm), se aprecia que existe diferencia significativa $(p<0,05)$ entre las medianas de cpm de los tres distritos, siendo mayor en Tarapoto y menor en Chiclayo. Con respecto al tiempo empleado

Tabla1. Características de los escolares de los colegios de los tres distritos incluidos en el estudio.

\begin{tabular}{|c|c|c|c|c|}
\hline \multirow[t]{2}{*}{ Características } & $\begin{array}{c}\text { Huaraz } \\
n=398\end{array}$ & $\begin{array}{c}\text { Chiclayo } \\
n=416\end{array}$ & $\begin{array}{c}\text { Tarapoto } \\
\mathrm{n}=427\end{array}$ & $\begin{array}{c}\text { Total } \\
n=1241\end{array}$ \\
\hline & $\mathrm{n}(\%)$ & $\mathrm{n}(\%)$ & $\mathrm{n}(\%)$ & $n(\%)$ \\
\hline \multicolumn{5}{|l|}{ Sexo } \\
\hline Masculino & $191(46,1)$ & $225(55,1)$ & $212(49,4)$ & $628(52,2)$ \\
\hline Femenino & $207(53,9)$ & $191(44,9)$ & $215(50,6)$ & $613(47,8)$ \\
\hline \multicolumn{5}{|l|}{ Edad (años) } \\
\hline 6 a 9 & $242(60,5)$ & $283(67,1)$ & $275(64,9)$ & $800(65,5)$ \\
\hline 10 a 13 & $156(39,5)$ & $133(32,9)$ & $152(35,1)$ & $441(34,5)$ \\
\hline \multicolumn{5}{|c|}{ Estado Nutricional } \\
\hline Delgadez & $4(1,0)$ & $3(0,7)$ & $1(0,2)$ & $8(0,6)$ \\
\hline Normal & $266(65,7)$ & $237(57,1)$ & $305(71,6)$ & $808(62,2)$ \\
\hline Sobrepeso & $82(21,9)$ & $106(25,1)$ & $66(15,5)$ & $254(22,1)$ \\
\hline Obeso & $46(11,4)$ & $70(17,1)$ & $55(12,7)$ & $171(15,1)$ \\
\hline
\end{tabular}

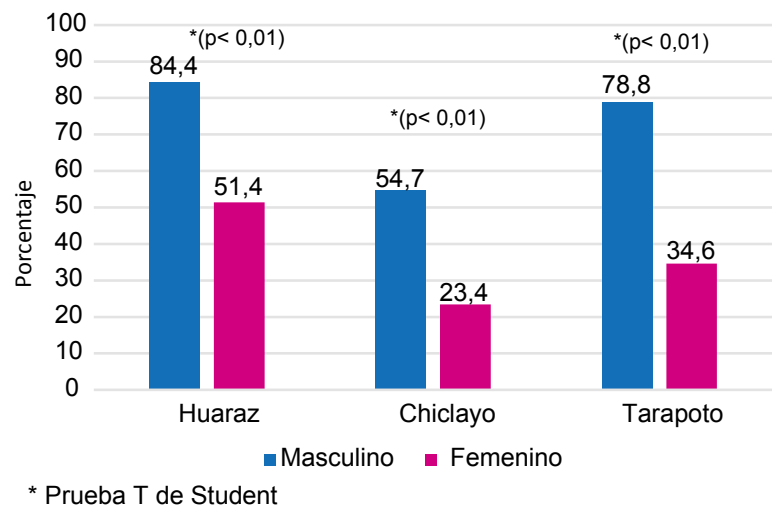

Figura 1. Porcentaje de cumplimiento de la recomendación de la OMS según sexo

en cada nivel de AF entre las 6 a 22 horas (periodo de vigilia en un día), se encontró que el tiempo invertido en actividades sedentarias durante el periodo de vigilia sobrepasa el $50 \%$ (más de 7 horas) y que el tiempo destinado a actividades ligeras corresponde a la tercera parte del periodo de vigilia (poco más de 4 horas), Así también las actividades moderadas representan el 6,2\% (alrededor de 50 minutos) y las intensas el 2,7\% (poco mas de 17 minutos) del periodo de vigilia de un día.

La Tabla 2 muestra la mediana de cuentas por minuto cpm y las medianas de los minutos de AFMI según sexo, edad y estado nutricional. Al respecto se encontró que los niños tienen un promedio de cpm más alto que las niñas en los tres ámbitos de estudio $(p<0,01)$. Además según edad se aprecia que la mediana/media de cpm es mayor en los niños de 6 a 9 años $(p<0,01)$ de las tres zonas. Por su parte según estado nutricional destaca que los escolares con sobrepeso y obesidad tienen un promedio de cpm ligeramente menor que los escolares con estado nutricional normal esta diferencia fue estadísticamente significativa solo en Huaraz $(p<0,01)$.

En cuanto al promedio de minutos de AFMI según sexo se encontró que los niños realizan más tiempo de AFMI que las niñas $(p<0,01)$ en los tres distritos. Según edad se encontró diferencia significativa $(p<0,05)$ entre las medianas de minutos de AFMI en Chiclayo y Tarapoto. Respecto al estado nutricional, los escolares de Chiclayo con sobrepeso y obesidad realizaron menos tiempo de AFMI que sus pares con estado nutricional normal $(p<0,01)$.

En la Tabla 3 se muestran los resultados del modelo de regresión lineal múltiple para los minutos de AFMI en todos los escolares. Se observa que según sexo los niños realizan 24 minutos más de AFMI en comparación con las niñas; según edad, los niños de 10 a 13 años 


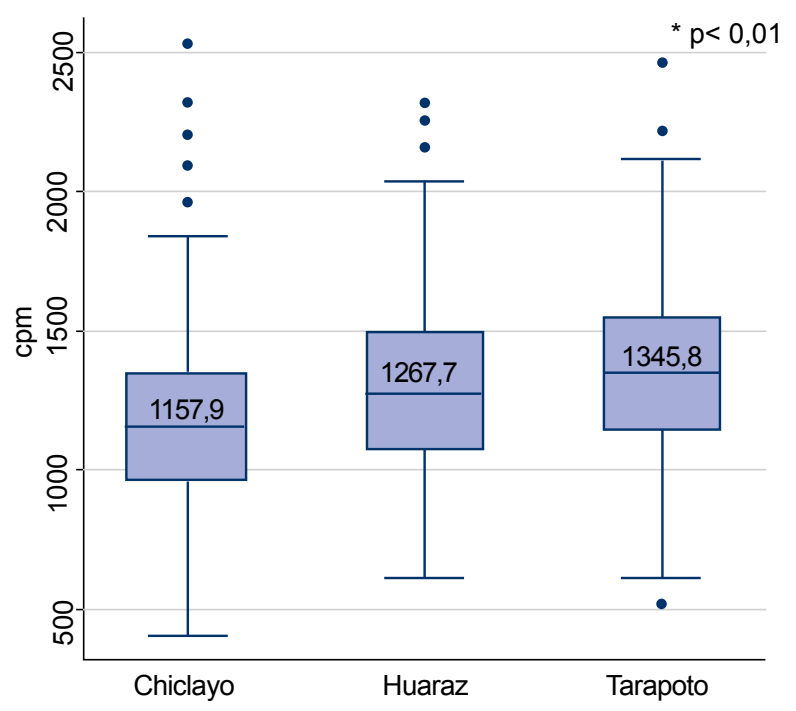

*Prueba Kruskal-Wallis

Figura 2. Mediana de las cuentas por minuto (cpm) en los escolares de los tres distritos considerados para el estudio

realizan menos minutos AFMI comparados con los de 6 a 9 años. El estado nutricional también influye sobre los minutos de AF, se aprecia que los escolares con obesidad realizan 13 minutos menos de AFMI en comparación con escolares normales o delgados. Asimismo, los escolares cuyas madres tienen un nivel educativo de secundaria o superior realizan menos minutos de AFMI respecto a los escolares con madres sin nivel educativo o primario. Al evaluar la influencia del distrito se encontró que los escolares de Chiclayo y Tarapoto, realizaban 8 y 17 minutos menos de AFMI, respectivamente, en comparación con los de Huaraz.

\section{DISCUSIÓN}

A nuestro conocimiento, este es el primer estudio en Perú que mide la AF con acelerómetros triaxiales, ello ha permitido determinar objetivamente la intensidad y tiempo de actividad física. Además, en comparación a los cuestionarios, la acelerometría entrega información sobre actividad física durante prácticamente la totalidad del tiempo de vigilia. Según recomienda la OMS en niños de 5 a 17 años se deben realizar 60 minutos al día como mínimo de AF moderada a vigorosa para obtener beneficios para la salud. Nuestros resultados muestran que alrededor del $50 \%$ de los escolares cumplen esta recomendación.

Considerando que un niño que cumple la recomendación tiene un nivel adecuado de AF, estos resultados guardan relación con otros estudios realizados en Perú. Así, Liria et al. ${ }^{\left({ }^{9}\right)}$ encontró que $48,2 \%$ de escolares tenían entre un buen nivel y un nivel regular de actividad física. Aparco et al. ${ }^{(10)}$ encontró un $72 \%$ de escolares entre los mismos niveles; mientras que Bustamante et al. (11) reporto un $67,5 \%$ de actividad física entre "moderadamente activos" y "muy activos". Si bien, las categorías de clasificación

Tabla 2. Cuantificación de la actividad física (AF) según sexo, edad y estado nutricional en escolares de los colegios de los tres distritos incluidos en el estudio

\begin{tabular}{|c|c|c|c|c|c|c|c|c|c|c|c|c|c|c|c|c|}
\hline & \multicolumn{4}{|c|}{ Huaraz } & \multicolumn{4}{|c|}{ Chiclayo } & \multicolumn{4}{|c|}{ Tarapoto } & \multicolumn{4}{|c|}{ Total } \\
\hline & \multicolumn{2}{|c|}{$\begin{array}{l}\text { AF medida en } \\
\text { cuentas por minuto }\end{array}$} & \multicolumn{2}{|c|}{$\begin{array}{c}\text { Minutos de } \\
\text { AF Moderada } \\
\text { intensa por día }\end{array}$} & \multicolumn{2}{|c|}{$\begin{array}{c}\text { AF medida en } \\
\text { cuentas por minuto }\end{array}$} & \multicolumn{2}{|c|}{$\begin{array}{c}\text { Minutos de } \\
\text { AF Moderada } \\
\text { intensa por día }\end{array}$} & \multicolumn{2}{|c|}{$\begin{array}{c}\text { AF medida en } \\
\text { cuentas por minuto }\end{array}$} & \multicolumn{2}{|c|}{$\begin{array}{c}\text { Minutos de AF } \\
\text { Moderada intensa } \\
\text { por día }\end{array}$} & \multicolumn{2}{|c|}{$\begin{array}{c}\text { AF medida en } \\
\text { cuentas por minuto }\end{array}$} & \multicolumn{2}{|c|}{$\begin{array}{c}\text { Minutos de } \\
\text { AF Moderada } \\
\text { intensa por día }\end{array}$} \\
\hline & $\begin{array}{l}\text { Mediana } \\
\text { (RIQ) }\end{array}$ & $\begin{array}{c}\text { valor } \\
\mathbf{p}\end{array}$ & $\begin{array}{l}\text { Mediana } \\
\text { (RIQ) }\end{array}$ & $\begin{array}{c}\text { valor } \\
p\end{array}$ & $\begin{array}{l}\text { Mediana } \\
\text { (RIQ) }\end{array}$ & $\begin{array}{c}\text { valor } \\
p\end{array}$ & $\begin{array}{c}\text { Mediana } \\
\text { (RIQ) }\end{array}$ & $\begin{array}{c}\text { valor } \\
p\end{array}$ & Media (DE) & $\begin{array}{c}\text { valor } \\
\mathbf{p}\end{array}$ & $\begin{array}{l}\text { Mediana } \\
\text { (RIQ) }\end{array}$ & $\begin{array}{c}\text { valor } \\
\mathbf{p}\end{array}$ & $\begin{array}{l}\text { Mediana } \\
\text { (RIQ) }\end{array}$ & $\begin{array}{c}\text { valor } \\
\mathbf{p}\end{array}$ & $\begin{array}{c}\text { Mediana } \\
\text { (RIQ) }\end{array}$ & $\begin{array}{c}\text { valor } \\
\mathbf{p}\end{array}$ \\
\hline Sexo * & & $<0,01$ & & $<0,01$ & & $<0,01$ & & $<0,01$ & & $<0,01$ & & $<0,01$ & & $<0,01$ & & $<0,01$ \\
\hline Masculino & $1417,3(362,9)$ & & $86,4(104,3)$ & & $1246,1(392,8)$ & & $62,8(28,3)$ & & $1462,6(19,2)$ & & $77,3(36,0)$ & & $1320,4(422,6)$ & & $68,5(34,1)$ & \\
\hline Femenino & $1160,9(346,3)$ & & $61,2(77,9)$ & & $1052,5(308,5)$ & & $43,8(25,8)$ & & $1233(20,6)$ & & $51,7(27,0)$ & & $1118,0(364,9)$ & & $50,0(27,2)$ & \\
\hline Edad (años) * & & $<0,01$ & & 0,21 & & $<0,01$ & & 0,046 & & $<0,01$ & & 0,02 & & $<0,01$ & & 0,02 \\
\hline 6 a 9 & $1354,0(401,6)$ & & $75,8(90,0)$ & & $1193,7(352,6)$ & & $56,5(29,3)$ & & $1409,0(17,6)$ & & $65,3(34,1)$ & & $1269,1(414,8)$ & & $60,8(31,4)$ & \\
\hline 10 a 13 & $1167,3(368,6)$ & & $67,8(89,3)$ & & $1052,5(364,6)$ & & $52,5(29,8)$ & & $1230,5(29,7)$ & & $60,1(39,1)$ & & $1118,2(397,1)$ & & $56,5(35,1)$ & \\
\hline Estado Nutricio & onal † & 0,047 & & 0,06 & & 0,09 & & $<0,01$ & & 0,9 & & 0,65 & & $<0,01$ & & $<0,01$ \\
\hline Delgadez & $870,8(433,8)$ & & $59,1(79,4)$ & & $1167,0(314,4)$ & & $66,6(18,0)$ & & $\ddagger$ & & $\ddagger$ & & $1167,0(314,4)$ & & $66,6(18,0)$ & \\
\hline Normal & $1304,8(439,8)$ & & $75,9(93,8)$ & & $1176,5(374,9)$ & & $59,2(28,7)$ & & $1345,7(21,3)$ & & $63,1(36,6)$ & & $1239,4(427,7)$ & & $61,8(33,6)$ & \\
\hline Sobrepeso & $1262,2(426,2)$ & & $70,3(88,4)$ & & $1145,0(396,8)$ & & $52,5(34,7)$ & & $1368,4(34,6)$ & & $67,3(32,6)$ & & $1200,1(418,0)$ & & $56,9(35,5)$ & \\
\hline Obeso & $1134,4(306,4)$ & & $65,3(81,9)$ & & $1068,3(372,8)$ & & $46,7(21,4)$ & & $1324(46,7)$ & & $63,6(37,3)$ & & $1133,4(348,5)$ & & $50,7(27,9)$ & \\
\hline
\end{tabular}

RIQ: Rango Intercuartilico.

* Prueba U de Mann-Whitney para ambas variables. Solo en Tarapoto se utilizó prueba t de student para las cuentas por minuto.

† Prueba de Kruskal-Wallis para ambas variables. Solo en Tarapoto se utilizó ANOVA para las cuentas por minuto.

¥ Solo se clasifico un escolar con delgadez en Tarapoto, cuyo valor de cuentas por minuto fue 1294,4 y tiempo de actividad física moderada intensa

fue de 54,2 minutos 
Tabla 3. Modelo de regresión lineal múltiple para los minutos de actividad física moderada intensa (AFMI)

\begin{tabular}{|c|c|c|}
\hline Variables & $\begin{array}{l}\text { Coeficiente } \beta \\
\text { (IC 95\%) }\end{array}$ & Valor $p$ \\
\hline \multicolumn{3}{|l|}{ Sexo } \\
\hline *Sexo masculino & $23,77(21,1$ a 26,5$)$ & $<0.01$ \\
\hline \multicolumn{3}{|l|}{ Edad(años) } \\
\hline †10-13 años & $-5,30(-1,7$ a $-8,9)$ & 0,01 \\
\hline \multicolumn{3}{|l|}{ IMC } \\
\hline ‡Sobrepeso & $-2,50(0,4$ a $-5,4)$ & 0,09 \\
\hline$\ddagger$ Obesidad & $-13,36(-9,1 \mathrm{a}-17,6)$ & $<0,01$ \\
\hline \multicolumn{3}{|l|}{ Educación de la madre } \\
\hline$\S$ Nivel secundaria & $-7,58(-2,5 \mathrm{a}-12,7)$ & $<0,01$ \\
\hline$\S$ Nivel superior & $-13,67(-8,1$ a $-19,2)$ & $<0,01$ \\
\hline \multicolumn{3}{|l|}{ Distritos } \\
\hline "Chiclayo & $-17,41(-12,8$ a $-22,0)$ & $<0,01$ \\
\hline ॥ Tarapoto & $-7,80(-3,7$ a $-11,9)$ & $<0,01$ \\
\hline
\end{tabular}

IC 95\%: intervalo de confianza al $95 \%$

*Comparado con sexo femenino

†Comparado con niños de 6 a 9 años

$\ddagger$ Comparado con niños delgados o normales

$\S$ Comparado con madres sin nivel educativo o solo primaria

|l Comparado con Huaraz

son diferentes, muestran un panorama de la realización de AF en escolares, similar a nuestros hallazgos.

En Sudamérica existen estudios con resultados similares, como en Argentina (17) donde el 45,4\% de niños y adolescentes registró niveles insuficientes de AF o en Colombia ${ }^{(18)}$ donde se encontró que $52,7 \%$ de los estudiantes no realizan ningún tipo de AF durante el reposo y permanecen sedentarios, el $27,2 \%$ practica $A F$ de moderada intensidad y el $20,1 \%$ practica AF vigorosa.

Además, nuestros resultados también son concordantes con lo reportado por el estudio americano de Troiano et al. ${ }^{(19)} \mathrm{y}$ al estudio europeo de Riddoch et al. (20) donde menos de la mitad de los niños entre 6 a 11 años cumplían con la recomendación OMS de actividad física. El cumplimiento de la recomendación de OMS es variable según sexo, nuestros resultados muestran que más varones que mujeres cumplen esta recomendación, como lo observado por Martínez et al. ${ }^{(21)}$ (35\% niños, 6\% niñas); Ruiz et al. ${ }^{(22)}$ (56,8\% niños, $27,5 \%$ niñas) y Konstabel et al. ${ }^{(23)}$.

Un aspecto a destacar en nuestro estudio es que para evaluar la intensidad de actividad física se utilizó el promedio de cuentas por minuto (cpm) con las categorías para niños de 6 a 10 años establecidos por Evenson et al. (13), considerando lo recomendado por una revisión sistemática ${ }^{24)}$ sobre los puntos de corte para AF moderada a intensa utilizado por varios autores. $A$ nivel internacional se han realizado estudios con acelerómetros, que son concordantes con nuestros hallazgos. Así, con respecto a la mediana de cpm se observó que los niños tuvieron una mediana mayor que las niñas $(p<0,01)$. Estas diferencias son reportadas por varios autores como Byrd ${ }^{(25)}$, y Mc Lure ${ }^{(26)}$ que describen un mayor promedio de cpm en varones con edades entre los cuatro a 10 años; y un estudio europeo que también reportó estas diferencias en adolescentes ${ }^{(22)}$.

Asimismo, el estudio encontró que a mayor edad disminuye la intensidad de $A F$, este hallazgo refrenda lo encontrado en el estudio IDEFICS ${ }^{(23)}$ en niños europeos que muestra una disminución de la actividad física a medida que avanza la edad, a partir de los 4 años. Al igual que un estudio sueco ${ }^{(27)}$, que indica que la $A F$ declina entre los 5 y 10 años.

La acelerometría ha permitido determinar, con mayor exactitud la cantidad de minutos al día empleados en actividades sedentarias, ligeras, moderadas y vigorosas. Encontramos que los escolares realizan actividades sedentarias más de la mitad del día y sumado a las actividades ligeras representan el $90 \%$ del día, mientras que las actividades moderadas solo alcanzan al $10 \%$ del tiempo. Estos datos son similares a lo encontrado por $\mathrm{Yu}$ et al. ${ }^{(28)}$ en escolares con sobrepeso quienes realizaban actividades sedentarias, en más del $51 \%$ de su tiempo. Así también Zamora et al. ${ }^{(29)}$ refiere un $72 \%$ de actividad sedentaria sin diferencia según estado nutricional.

Con respecto al tiempo dedicado a la AFMI, nuestros resultados muestran una marcada diferencia según género $(p<0,01)$, en los tres distritos. Esto es similar a lo reportado por Noel et al. ${ }^{(30)}$ con 28,4 minutos en varones versus 19,5 minutos en mujeres de AFMI $(p<0,01)$ y Martínez et al. (21) que observó que los varones presentaban más minutos de actividad física vigorosa que las mujeres.

El estudio tiene algunas limitaciones ligadas al uso de acelerómetros, como el no considerar las actividades que se realizan en el agua, ya que la recomendación es quitarse el acelerómetro durante estas actividades; además se observó que el $7 \%$ de niños en Chiclayo y 9,5\% Tarapoto modificaron sus hábitos de actividad física al usar el acelerómetro. Otra limitación del método es registrar periodos con información incompleta, cuando los usuarios se quitan los acelerómetros; en el estudio se recomendó a los escolares su uso durante 7 días, sin embargo, hubieron niños que completaron información para un período menor a 7 días; al respecto diversos estudios analizan datos con períodos mínimos 3 días con información completa ${ }^{(22,23)}$. Adicionalmente se debe precisar que el estudio se hizo en las zonas urbanas de tres distritos y no se puede hacer inferencias a todo el Perú, sin embargo, los datos encontrados reflejan la 
realidad de la actividad física en los escolares de las zonas de estudio.

En conclusión, cerca del $50 \%$ de escolares cumple la recomendación de OMS sobre actividad física diaria. Se evidencia que los niños son más activos que las niñas, además que a mayor edad los escolares son menos activos. Según estado nutricional los escolares con obesidad realizan menos AF que aquellos que están con peso saludable.

La prevención del sobrepeso y obesidad en niños en edad escolar debería basarse en intervenciones integrales: promover una alimentación saludable tanto en el hogar como en la escuela; motivar el desarrollo de la AF enfocada en cumplir la recomendación OMS, así como disminuir el tiempo de actividades sedentarias con el fin de obtener beneficios para la salud. Además se debe tener en cuenta las diferencias de AF según género y edad para el diseño de las intervenciones.
Los gobiernos locales deben garantizar espacios públicos apropiados para la realización deAF, desarrollar legislación y políticas públicas orientadas a incentivar estilos de vida saludable, regular la información y la publicidad sobre alimentos y bebidas dirigidas especialmente a los niños. Finalmente se recomienda la medición objetiva de la AF en estudios posteriores, no solo para precisar el estado de la población sino también para obtener una medida más fiable de la efectividad de las intervenciones.

Contribuciones de autoría: KA, LH, JPA han participado en la concepción y diseño del artículo. KA, JP participaron en la recolección, análisis en interpretación de datos. Todos los autores participaron en la redacción del borrador de artículo, revisaron críticamente el artículo y aprobaron la versión final.

Fuente de financiamiento: el estudio fue financiado por el Instituto Nacional de Salud de Perú.

Conflictos de interés: los autores declaran no tener conflictos de interés.

\section{REFERENCIAS BIBLIOGRÁFICAS}

1. World Health Organization. Global health risks: mortality and burden of disease attributable to selected major risks. [Internet] Geneva: WHO; 2009 [citado el 12 de julio de 2016]. Disponible en: http://www.who.int/ healthinfo/global_burden_disease/ GlobalHealthRisks_report_full.pdf.

2. Veiga O, Martinez-Gomez D. Beneficios de la actividad física en la infancia y la adolescencia. En: Universidad San Pablo. Nutrición, Vida activa y Deporte. 1ra ed. Madrid: International Marketing and comunication; 2010. p. 67-80

3. Caspersen CJ, Powell KE, Christenson GM. Physical Activity, Exercise, and Physical Fitness: Definitions and Distinctions for HealthRelated Research. Public Health Rep.1985;100(2):126-31.

4. Ojeda R, Navarro CM. Análisis de la frecuencia y niveles de intensidad en la actividad física realizada por adolescentes mediante acelerometría. En: VII Congreso Nacional de Ciencias del Deporte y la Educación Física. Pontevedra, España: Universidad de Vigo; 2011.

5. Janssen I, LeBlanc AG. Systematic review of the health benefits of physical activity and fitness in schoolaged children and youth. Int J Behav Nutr Phys Act. 2010;7:40. doi: 10.1186/1479-5868-7-40.
6. Corbin CB, Pangrazi RP, Masurier GCL. Physical activity for Children: Current Patterns and Guidelines. J Phys Act Health. 2004;1(3):281. doi: 10.1123/jpah.1.3.281

7. Hu F. Obesity Epidemiology. New York: Oxford University Press, Inc.; 2008.

8. Rodríguez Ordax J, Terrados $\mathrm{N}$. Métodos para la valoración de la actividad física y el gasto energético en niños y adultos. Arch med deporte. 2006; 23(115):365-77.

9. Liria MR, Mispireta ML, Lanata CF, Creed-Kanashiro HM. Perfil Nutricional en escolares de Lima y Callao. Lima: Instituto de Investigación Nutricional, 2008.

10. Aparco JP, Bautista-Olórtegui W, Astete-Robilliard L, Pillaca J. Evaluación del estado nutricional, patrones de consumo alimentario y de actividad física en escolares del Cercado de Lima. Rev Peru Med Exp Salud Pública. 2016; 33(4):633-9. doi: 10.17843/rpmesp.2016.334.2545.

11. Bustamante A, Seabra AF, Garganta RM, Maia JA Efectos de la actividad física y del nivel socioeconómico en el sobrepeso y obesidad de escolares Lima Este 2005. Rev Perú Med Exp Salud Pública. 2007;24(2):121-128.

12. Calahorro F, Torres-Luque G, LópezFernández I, Santos-Lozano A,
Garatachea N, Álvarez E. Actividad física y acelerometría; orientaciones metodológicas, recomendaciones y patrones. Nutr Hosp. 2015;31(1):11528. doi: 10.3305/nh.2015.31.1.7450.

13. Evenson KR, Catellier DJ, Gill $\mathrm{K}$, Ondrak KS, McMurray RG. Calibration of two objective measures of physical activity for children. J Sports Sci.2008;26(14):1557-65. doi: $10.1080 / 02640410802334196$

14. Organización Mundial de la Salud. Recomendaciones mundiales sobre actividad física para la salud. Publicaciones de la Estrategia mundial sobre régimen alimentario, actividad física y salud. Ginebra: OMS; 2010.

15. World Health Organization. Growth reference 5-19 years: WHO AnthroPlus software [Internet]. Geneva: WHO; c2017 [citado el 12 de julio de 2016]. Disponible en: http://www.who.int/ growthref/tools/en/

16. World Health Organization. Growth reference 5-19 years: BMI-for-age (519 years) [Internet]. Geneva: WHO; c2017 [citado el 12 de julio de 2016]. Disponible en: http://www.who.int/ growthref/who2007_bmi_for_age/en/

17. Tuñón I, Laíño F. Insuficiente actividad física en la infancia. Niños, niñas y adolescentes entre 5 y 17 años en la Argentina urbana $1^{\text {a }}$ ed. Buenos Aires: Educa; 2014. 
18. Arias Arias EA. Niveles de actividad física de niños y adolescentes durante el descanso en la escuela, un estudio observacional con el uso de SOPLAY. Educ fis deport. 2014; 33(1): 175-91.

19. Troiano RP, Berringan D, Dodd KW, Masse LC, Tilert T, McDowell M. Physical activity in the United states measured by accelerometer. Med Sci Sports Exerc 2008;40(1):181-8. doi: $10.1249 /$ mss.0b013e31815a51b3.

20. Riddoch CJ, Mattocks C, Deere K, Saunders J, Kirkby J, Tilling K, et al. Objective measurement of levels and patterns of physical activity. Arch Dis Child 2007:92(11):963-9. doi: 10.1136/adc.2006.112136.

21. Martínez Martínez J, Contreras Jordán OR, Aznar Laín S, Lera Navarro A. Niveles de actividad física medido con acelerómetro en alumnos de $3^{\circ}$ ciclo de Educación Primaria: actividad física diaria y sesiones de Educación Física. Revista de Psicología del Deporte. 2012;21(1):117-23.

22. Ruiz JR, Ortega FB, MartinezGomez D, Labayen I, Moreno LA, De Bourdeaudhuij I et al. Objectively measured physical activity and sedentary time in European adolescents: the HELENA study. Am
J Epidemiol. 2011;174(2):173-84. doi: 10.1093/aje/kwr068.

23. Konstabel K, Veidebaum T, Verbestel V, Moreno LA, Bammann K, Tornaritis $\mathrm{M}$, et al. Objectively measured physical activity in European children: The IDEFICS Study. Int J Obes 2014;38 Suppl 2: 135-43. doi: 10.1038/ ijo.2014.144.

24. Kim Y, Beets M, Welk G. Everything you wanted to know about selecting the "right" Actigraph accelerometer cut-points for youth, but...: A systematic review. J Sci Med Sport 2012; 15(4): 311-21. doi: 10.1016/j. jsams.2011.12.001.

25. Byrd-Williams C, Kelly LA, Davis JN, Spruijt-Metz D, Goran MI. Influence of gender, BMI and Hispanic ethnicity on physical activity in children. Int Jour Pediatric Obes. 2007; 2(3):159-66. doi: 10.1080/17477160701369167

26. McLure SA, Summerbell CD, Reilly JJ. Objectively measured habitual physical activity in a highly obesogenic environment. Child Care Health Dev. 2009;35(3):369-75. doi: 10.1111/j.1365-2214.2009.00946.x

27. Nyberg GA, Nordenfelt AM, Ekelund U, Marcus C. Physical activity patterns measured by accelerometry in 6- to 10-year-old children. Med Sci Sports Exerc. 2009; $41(10): 1842-8$. doi: 10.1249/MSS.0b013e3181a48ee6.

28. Yu CW, Sung RY, So R, Lam K, Nelson EA, Li AM, et al. Energy expenditure and physical activity of obese children: cross-sectional study. Hong Kong Med J. 2002;8(5):313-7.

29. Zamora JD, Laclé A. Evaluación del gasto energético y actividad física en escolares eutróficos, con sobrepeso u obesidad. Rev chil pediatr 2012;83(2):134-45.

30. Noel SE, Mattocks C, Emmett P, Riddoch CJ, Ness AR, Newby PK. Use of accelerometer data in prediction equations for capturing implausible dietary intakes in adolescents. Am J Clin Nutr. 2010;92(6):1436-45. doi: 10.3945/ajcn.2010.29386.

Correspondencia: Katherine Alvis Chirinos Dirección: Av. Tizón y Bueno 276 Jesús Maria, Perú

Teléfono: (511) 948961605

Correo electrónico:kalvis@ins.gob.pe

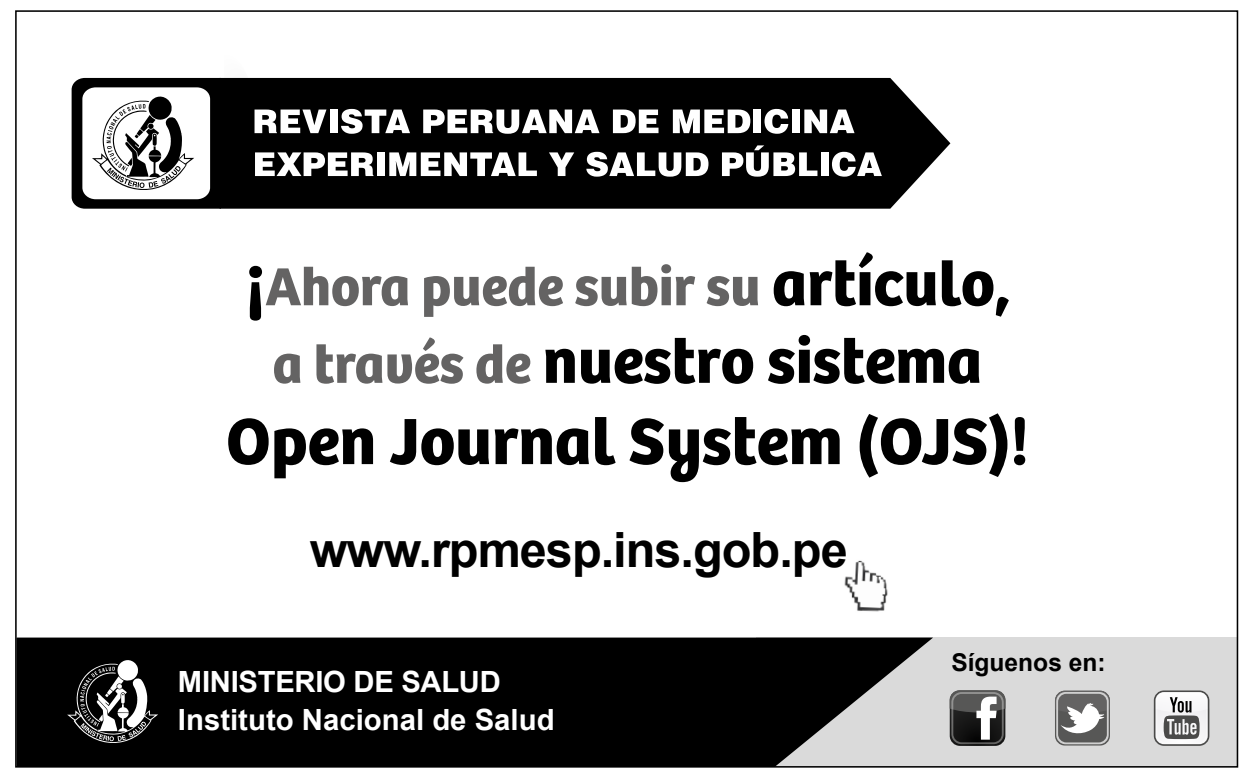

